\title{
Le Mouillage
}

\section{Introduction}

Les premières études sur le mouillage d'un solide par un fluide remontent au XIX $\mathrm{X}^{\mathrm{e}}$ siècle, aux pionniers de la capillarité. Une deuxième vague a été lancée par Zisman à l'Office of Naval Research, et une autre par l'école de Deyagin en U.R.S.S. Plus récemment des apports d'origine très différente ont activé la recherche : (1) l'intérêt des compagnies pétrolières pour la récupération assistée, intérêt malheureusement variable avec les cours du brut, mais qui a joué un rôle de catalyseur non négligeable en France ; (2) l'intérêt des métallurgistes, cristallisé par les prédicteurs remarquables de Cahn concernant la transition de mouillage ; (3) le perfectionnement des systèmes modèles, en particulier par l'apport de la microélectronique qui permet d'engendrer des surfaces admirables, et par le développement des traitements chimiques de surface ; (4) la théorie, elle aussi; a progressé, malgré le constant hiatus entre mécaniciens et physiciens.

Les articles rassemblés ici par J.-F. Joanny illustrent bien ces différents aspects. Certains concernent la capillarité au sens le plus classique : gouttes, ferro-fluides, comportement sur un support mince ou sur une fibre. D'autres sont plus concernés par l'effet des forces à longue portée, la revue de N. Churaev, décrivant toute la perspective de l'école soviétique, est particulièrement intéressante à cet égard. D'autres encore portent sur des effets de surface aléatoires : milieux poreux ou surfaces rugueuses. Les propriétés à l'échelon chimique sont sous-développées, et je le regrette un peu, mais le lecteur trouvera quand même dans ce secteur une discussion vivante de l'interface métal/oxyde. Au total, ce fascicule ne couvre, certes, pas tout le domaine, mais il donne une vision actuelle et raisonnée de quelques problèmes majeurs : il sera, je pense, très utile.

P. G. de Gennes 